\title{
Structural and Electrical Properties of SiC Grown by PVT Method in the Presence of the Cerium Vapor
}

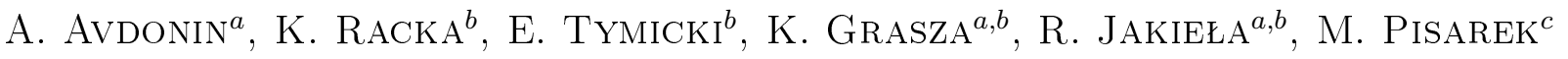 \\ AND W. DOBROWOLSKI ${ }^{a}$ \\ ${ }^{a}$ Institute of Physics, Polish Academy of Sciences, al. Lotników 32/46, 02-668 Warsaw, Poland \\ ${ }^{b}$ Institute of Electronic Materials Technology, Wólczyńska 133, 01-919 Warsaw, Poland \\ ${ }^{c}$ Institute of Physical Chemistry, PAS, M. Kasprzaka 44/52, 01-224 Warsaw, Poland
}

\begin{abstract}
The results of investigation of structural and electrical properties of bulk SiC crystals, which were grown by physical vapor transport method with different $\mathrm{Ce}$ impurity content added to the $\mathrm{SiC}$ source material, are presented. The gradual dosage of cerium from the $\mathrm{SiC}$ source and continuous presence of the cerium vapor over the $\mathrm{SiC}$ crystallization fronts during the crystal growth processes are confirmed. The cerium influences the overall concentration of structural defects. The increase of the concentration of both, donors and acceptors, and appearance of new shallow donors $(15-32 \mathrm{meV})$ in $4 \mathrm{H}-\mathrm{SiC}$ crystals are observed.
\end{abstract}

DOI: 10.12693/APhysPolA.124.761

PACS: 74.25.F-, 61.72.-y, 81.10.Bk, 61.72.uf

\section{Introduction}

Rare-earth ion doped semiconductors are usually investigated as a potential candidate for promising applications, such as phosphors, scintillators or solid-state lasers [1-3]. Many reports concern the investigations of materials doped with optically active $\mathrm{Ce}^{3+}$ ions, e.g. YAG:Ce, $\mathrm{Lu}_{2} \mathrm{SiO}_{5}$ :Ce, Ce-doped fluoride crystals, but there is a lack of data concerning the Ce-doping of $\mathrm{SiC}$. There is only one paper by Itoh et al. [4], in which, for bulk $4 \mathrm{H}-\mathrm{SiC}$ crystals grown by physical vapor transport (PVT) method in the presence of the cerium impurity, the non-uniform concentrations of Ce atoms at levels less than $10^{17} \mathrm{~cm}^{-3}$ (which was a detection limit in the secondary ion mass spectroscopy (SIMS) measurements) up to $10^{20} \mathrm{~cm}^{-3}$ are reported.

In this work, we present the results of investigations of bulk $\mathrm{SiC}$ crystals, which were grown by PVT method in the presence of the cerium vapor. Our goal was to study the effect of possible cerium incorporation into the crystalline lattice of $\mathrm{SiC}$ with its consequences on structural and electrical properties of $\mathrm{SiC}$.

\section{Experimental}

Bulk $\mathrm{SiC}$ crystals were grown by PVT method on the $\mathrm{C}$-face or the Si-face of the crystal seeds with different $\mathrm{Ce}$ impurity content ( $0.1 \mathrm{wt} \%, 1.0 \mathrm{wt} \%$, and $5.0 \mathrm{wt} \%)$ added to the $\mathrm{SiC}$ source material. As the source of cerium, a commercial granular $\mathrm{CeSi}_{2}$ was used. The reference $\mathrm{SiC}$ crystal, which was grown without the Ce additive and with the same growth parameters was also studied. Structural properties were studied by X-ray diffraction (XRD), X-ray photoemission spectroscopy (XPS), and SIMS. The Hall effect and electrical conductivity of the samples were measured in the van der Pauw geometry, with four electrical contacts placed on the top side (perpendicular to $c$-axis) in the corners of a square sample, with dimensions of $4 \times 4 \times 1 \mathrm{~mm}^{3}$. Electrical contacts were made by vacuum deposition of $\mathrm{Ni}$ followed by thermal treatment at $900^{\circ} \mathrm{C}$ for $5 \mathrm{~min}$. The measuring leads were attached to Ni contact pads with silver conductive paste. Measurements were carried out in the temperature range between $4.5 \mathrm{~K}$ and $500 \mathrm{~K}$, and in the magnetic field of $1 \mathrm{~T}$. Usually, below $70-100 \mathrm{~K}$ the samples could not be measured, because the quality of Ni contacts rapidly decreased with decreasing temperature. Even though the concentration of carriers and conductivity were high enough, the continuation of measurements was impossible because of increasing electrical noise, which was most evident in the measurement of the Hall voltage. At low temperatures (around $70 \mathrm{~K}$ ), in many samples, the noise had a distinct three-level structure. The noise is attributed to non-linearity of the $I(V)$ characteristics of the Ni contacts at low temperatures, which act as the Schottky diodes and detect the surrounding electromagnetic waves. Carrying out the experiment in this temperature range, despite the effects of noise, causes the unexplained increase of the measured free carrier concentration with decreasing temperature.

\section{Results and discussion}

The $\mathrm{SiC}$ crystals, which were grown in the presence of $1 \mathrm{wt} \% \mathrm{Ce}$ or $5 \mathrm{wt} \% \mathrm{Ce}$ on the C-face of the crystal seed were of the $4 \mathrm{H}-\mathrm{SiC}$ polytype, while the crystal grown with $0.1 \mathrm{wt} \%$ Ce on the Si-face of the crystal seed was of the $6 \mathrm{H}-\mathrm{SiC}$ polytype. The undoped $\mathrm{SiC}$ reference crystal $(0 \mathrm{wt} \% \mathrm{Ce}$ ) grown on the $\mathrm{C}$-face of the crystal seed was also of the $6 \mathrm{H}-\mathrm{SiC}$ polytype. This was confirmed by XRD (spectra are not shown here). Typical values of the lattice constants were: $a=3.08 \AA, c=10.08 \AA$ for $4 \mathrm{H}-\mathrm{SiC}$, and $a=3.08 \AA, c=15.11 \AA$ for $6 \mathrm{H}-\mathrm{SiC}$.

The Ce atoms do not seem to incorporate in the $\mathrm{SiC}$ crystal lattice like ordinary impurities. For all $\mathrm{SiC}$ crystals grown in the presence of cerium impurity, a mixture 


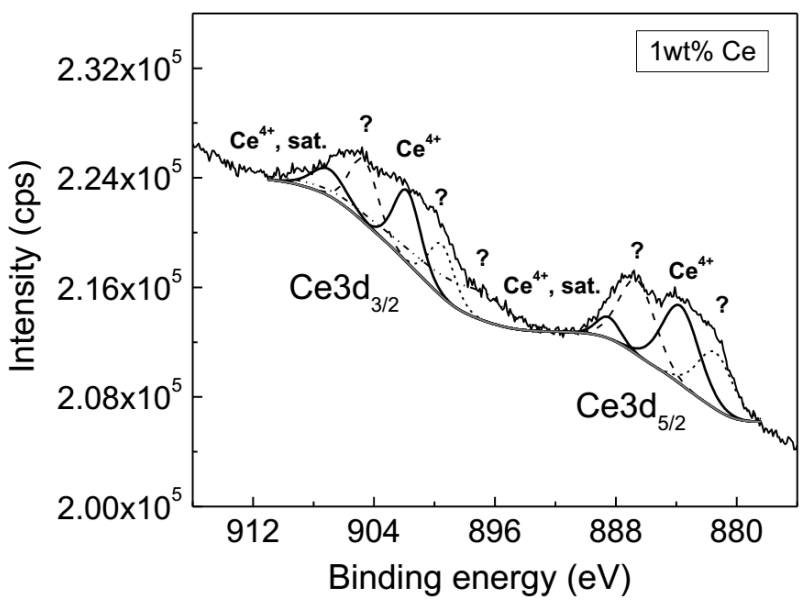

Fig. 1. Ce high-resolution XPS spectrum recorded for crystallization front of $1 \mathrm{wt} \%$ Ce sample, and its decomposition into components related to the cerium oxide $\mathrm{CeO}_{2}$ (the satellite peaks of $\mathrm{CeO}_{2}$ are denoted by $\mathrm{Ce}^{4+}$, sat.) and some unidentified Ce-species (the peaks denoted by "?").

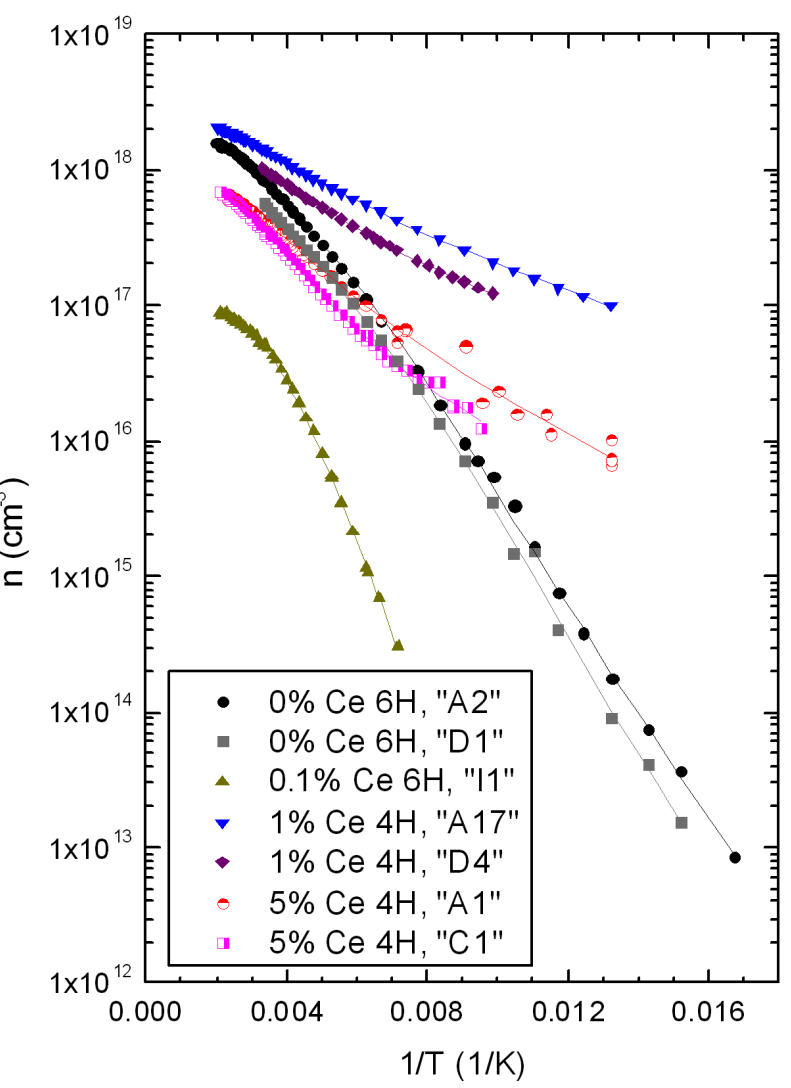

Fig. 2. Temperature dependence of electron concentration (dots) for $\mathrm{SiC}$ samples grown in the presence of different Ce impurity content. Lines represent fitted dependences, obtained using parameters from Table.

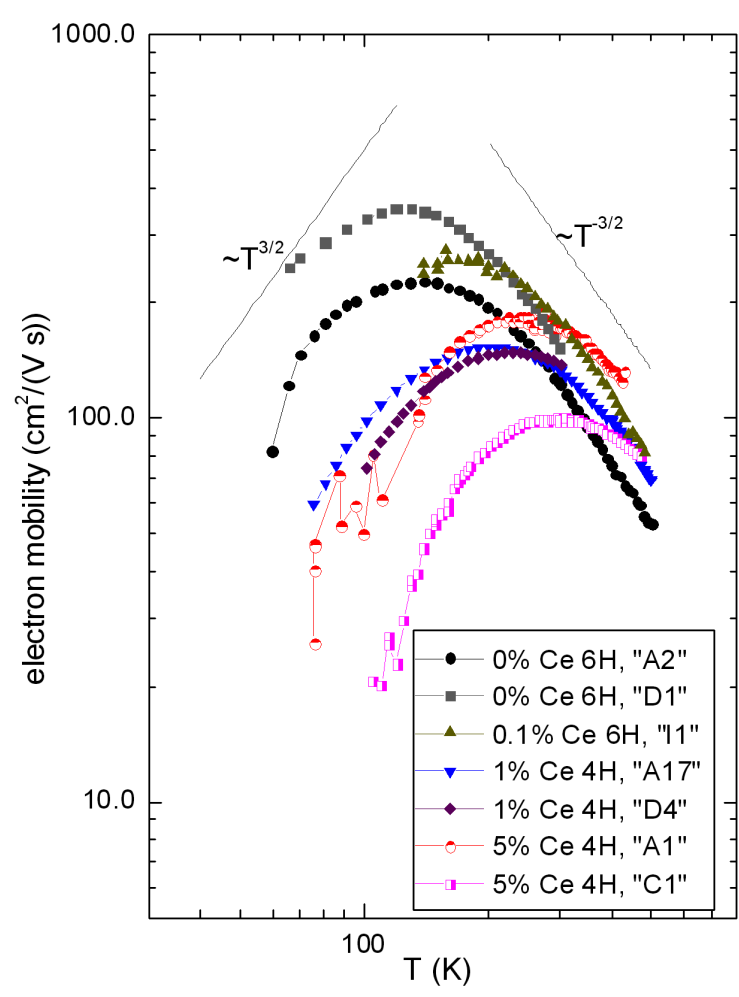

Fig. 3. Temperature dependence of electron mobility for $\mathrm{SiC}$ samples grown in the presence of different $\mathrm{Ce}$ impurity content.

of $\mathrm{CeO}_{2}$ and some unidentified (at this stage) Ce-species was found by XPS measurements only on the $\mathrm{SiC}$ crystallization fronts (Fig. 1), which points out the condensation of cerium vapor species at these fronts upon cooling-crystallization process, after the effective crystal growth stage.

All measured $\mathrm{SiC}$ samples, which were cut from the bulk of $\mathrm{SiC}$, were found to be of the $n$-type of the electrical conductivity. The measured dependences of electron concentration and the electron Hall mobility on temperature, $n(T)$ and $\mu(T)$, are shown in Fig. 2 and Fig. 3, respectively. Both the mobility and the electron concentration were calculated with the assumption that the electron scattering factor is equal to 1 . The temperature dependence of the electron concentration has activational character, and was used to evaluate the parameters of the crystal point defects responsible for the observed conductivity. The parameters were obtained by fitting the solution of the electrical neutrality equation [5] to the experimental $n(T)$ dependence. In most fits two shallow donor levels were assumed in the model, because within a single donor model the experimental dependence could not be fitted, or the two donor model provided a better fit. In case of two donor model the electrical neutrality equation was solved numerically. A model with three donor levels was also applied to fit the experimental data; however, in this case the third level was always deep and 
did not improve the quality of the fit. Within the model with two shallow donor levels, five fitting parameters were used (i.e. activation energies, concentrations of the donor defects and the concentration of compensating acceptor defects). The material parameters used in calculation are as follows: free electron effective masses: $m^{*}=0.27$ (for $6 \mathrm{H}-\mathrm{SiC}$ ) and $m^{*}=0.19$ (for $4 \mathrm{H}-\mathrm{SiC}$ ) [6], number of equivalent conductance band minima: 6 , and the spin degeneracy of donor levels: 2 .

The results of the fitting procedure are summarized in Table where: $E_{\mathrm{d} 1}$ and $N_{\mathrm{d} 1}$ parameters represent the activation energy and concentration of the first (shallower) donor level respectively; $E_{\mathrm{d} 2}$ and $N_{\mathrm{d} 2}$ are the activation energy and concentration of the second (deeper) donor level, and $N_{\mathrm{a}}$ is the concentration of compensating acceptors in the two-donor-level model. The curves obtained using the fitting parameters mentioned above are shown in Fig. 2 (lines) along with the experimental data (dots). The more reliable values (i.e. obtained by fitting the data within wider temperature ranges) are marked with bold characters in Table. Since the nitrogen impu- rity was one of the common impurities and a dominant dopant in the $\mathrm{SiC}$ source material used in our $\mathrm{SiC}$ growth processes, and since the concentration of Ce atoms in the measured samples was at levels below a detection limit in SIMS measurements, we are inclined to attribute the observed defects to substitutional nitrogen donors. The nitrogen-related defects in $\mathrm{SiC}$ are relatively well known; however, the parameters of such defects are a matter of discussion. Nitrogen in $\mathrm{SiC}$ is believed to create two types of donor defects, depending on the lattice site, in which it substitutes the carbon atom. Cubic-site and hexagonal-site substitutional nitrogen donors are distinguished. In the $6 \mathrm{H}-\mathrm{SiC}$ polytype such defects have activation energies of $144 \mathrm{meV}$ and $80 \mathrm{meV}$, respectively [7, 8]. There are two different cubic sites in $6 \mathrm{H}-\mathrm{SiC}$, however the difference in the activation energy of these defects cannot be distinguished by the Hall effect measurements. On the other hand, in the $4 \mathrm{H}-\mathrm{SiC}$ polytype the same defects are shown to have slightly smaller activation energies of 120 $130 \mathrm{meV}$ and 50-60 meV, respectively ([8] and references therein).

\section{TABLE}

The parameters of the $\mathrm{SiC}$ crystal point defects obtained by fitting the solution of the electrical neutrality equation to the experimental $n(T)$ dependences. The more reliable values are marked with bold characters. The second column shows the polarization of the crystal seed, on which $\mathrm{SiC}$ crystals were grown.

\begin{tabular}{c|c|c|c|c|c|c}
\hline \hline Sample & $\begin{array}{c}\text { Crystal seed } \\
\text { polarization }\end{array}$ & $\begin{array}{c}E_{\mathrm{d} 1} \\
{[\mathrm{meV}]}\end{array}$ & $\begin{array}{c}N_{\mathrm{d} 1} \\
{\left[\mathrm{~cm}^{-3}\right]}\end{array}$ & $\begin{array}{c}E_{\mathrm{d} 2} \\
{[\mathrm{meV}]}\end{array}$ & $\begin{array}{c}N_{\mathrm{d} 2} \\
{\left[\mathrm{~cm}^{-3}\right]}\end{array}$ & $\begin{array}{c}N_{\mathrm{a}} \\
{\left[\mathrm{cm}^{-3}\right]}\end{array}$ \\
\hline $0 \% \mathrm{Ce}(\mathrm{A} 2) ; 6 \mathrm{H}$ & C-face & $\mathbf{6 9}$ & $\mathbf{2 . 7} \times \mathbf{1 0}^{\mathbf{1 8}}$ & - & - & $\mathbf{4 . 1} \times \mathbf{1 0}^{\mathbf{1 7}}$ \\
$0 \% \mathrm{Ce}(\mathrm{D} 1) ; 6 \mathrm{H}$ & C-face & 76 & $1.3 \times 10^{18}$ & & & $1.3 \times 10^{17}$ \\
$0.1 \% \mathrm{Ce}(\mathrm{I} 1) ; 6 \mathrm{H}$ & Si-face & $\mathbf{1 1 9}$ & $\mathbf{1 . 6} \times \mathbf{1 0}^{\mathbf{1 7}}$ & - & - & $\mathbf{5 . 9} \times \mathbf{1 0}^{\mathbf{1 6}}$ \\
$1 \% \mathrm{Ce}(\mathrm{A} 17) ; 4 \mathrm{H}$ & C-face & $\mathbf{1 1}$ & $\mathbf{8 . 3} \times \mathbf{1 0}^{\mathbf{1 7}}$ & $\mathbf{4 3}$ & $\mathbf{2 . 6} \times \mathbf{1 0}^{\mathbf{1 8}}$ & $\mathbf{4 . 1} \times \mathbf{1 0}^{\mathbf{1 7}}$ \\
$1 \% \mathrm{Ce}(\mathrm{D} 4) ; 4 \mathrm{H}$ & C-face & 17 & $7.2 \times 10^{17}$ & 60 & $2.1 \times 10^{18}$ & $3.4 \times 10^{17}$ \\
$5 \% \mathrm{Ce}(\mathrm{A} 1) ; 4 \mathrm{H}$ & C-face & $\mathbf{1 9}$ & $\mathbf{1 . 1} \times \mathbf{1 0}^{\mathbf{1 8}}$ & $\mathbf{7 4}$ & $\mathbf{8 . 3} \times \mathbf{1 0}^{\mathbf{1 7}}$ & $\mathbf{9 . 6} \times \mathbf{1 0}^{\mathbf{1 7}}$ \\
$5 \% \mathrm{Ce}(\mathrm{C} 1) ; 4 \mathrm{H}$ & C-face & 17 & $9.8 \times 10^{17}$ & 93 & $1.1 \times 10^{18}$ & $9.0 \times 10^{17}$
\end{tabular}

The samples, which were grown without Ce impurity $(0 \mathrm{wt} \% \mathrm{Ce}$ ) were of the $6 \mathrm{H}-\mathrm{SiC}$ polytype. For these samples the obtained donor activation energies are $69 \mathrm{meV}$ and $76 \mathrm{meV}$ (see Table). This donor defect corresponds well to the previously reported value of the energy level of the nitrogen donor [6].

The concentration of Ce in the growth chamber had a strong influence on the content of point defects in the grown $\mathrm{SiC}$ crystals. The $6 \mathrm{H}-\mathrm{SiC}$ sample, which was grown on the Si-face of the crystal seed and in the presence of only $0.1 \mathrm{wt} \% \mathrm{Ce}$ is heavily compensated, and its most shallow active donor level has the energy of $119 \mathrm{meV}$, which is also close to reported in the literature nitrogen impurity level $[7,8]$. In $\mathrm{SiC}$ samples, which were grown in the presence of higher concentration of $\mathrm{Ce}$ (1 wt\% or $5 \mathrm{wt} \%$ ) the concentration of free carriers is an order of magnitude higher than for the $0.1 \mathrm{wt} \% \mathrm{Ce}$ sample, and the activation energy of the donors is much smaller in the range of $11-19 \mathrm{meV}$ (see Table). Such shallow levels to our knowledge were not observed in nitrogen doped $4 \mathrm{H}-\mathrm{SiC}$. For these samples, the fitting procedure with the one-level model was impossible to achieve. For $1 \mathrm{wt} \% \mathrm{Ce}$ sample the next energy level of $\approx 43-60 \mathrm{meV}$ corresponds well to the energy of nitrogen defect in $4 \mathrm{H}-\mathrm{SiC}$ [8]; however, for $5 \mathrm{wt} \%$ Ce samples we have obtained a deeper energy level of $\approx 74 \mathrm{meV}$. This value does not correspond exactly to the nitrogen level in $4 \mathrm{H}-\mathrm{SiC}$, instead it is closer to the level of $\mathrm{N}$-donor in $6 \mathrm{H}-\mathrm{SiC}$.

The structural investigations show that Ce atoms do not enter the bulk of the $\mathrm{SiC}$ crystals. However, there is no doubt that presence of $\mathrm{Ce}$ in the growth atmosphere stabilizes the growth of the $4 \mathrm{H}$ polytype for crystals grown on the $\mathrm{C}$-face of the crystal seed. In the same time, we believe that the presence of $\mathrm{Ce}$ in the growth chamber and on the crystal surface changes the composi- 
tion of defects in the growing crystal. We do not have yet the information, which would point at the microscopic structure of shallow $(\approx 20 \mathrm{meV})$ defects, but our hypothesis is that these defects are possibly some complex defects consisting of substitutional nitrogen defect with some native defects, which form in the presence of $\mathrm{Ce}$ impurity.

There is a possibility that such shallow $(\approx 20 \mathrm{meV})$ defects existed in all samples, but they were not observed due to compensation, and in $1 \mathrm{wt} \% \mathrm{Ce}$ and $5 \mathrm{wt} \% \mathrm{Ce}$ samples these levels are visible because the compensation in these samples is weak. The temperature dependence of the mobility (Fig. 3) shows that in $1 \mathrm{wt} \% \mathrm{Ce}$ and $5 \mathrm{wt} \%$ Ce samples the concentration of ionized impurities is much higher than in $\mathrm{SiC}$ samples with $0 \mathrm{wt} \% \mathrm{Ce}$, and it increases with the increase of Ce content in the $\mathrm{SiC}$ source material (in the growth chamber). This is confirmed by the facts that with increasing content of Ce: (i) the carrier mobility in the samples decreases, and (ii) that the maximum of the mobility vs. temperature curve shifts to higher temperatures. Thus, under influence of Ce in the growth chamber, the overall concentration of defects increases for both, donors and acceptors, and the new shallow nitrogen-related donors appear in $4 \mathrm{H}-\mathrm{SiC}$. A similar conclusion was reached in [9], where author assigned defects with activation energy of $\approx 20 \mathrm{meV}$ to negatively charged $D^{-}$donors or complex defects.

\section{Conclusions}

For $\mathrm{SiC}$ crystals, which were grown on the $\mathrm{C}$-face of the crystal seed, the presence of Ce impurity in the growth atmosphere stabilized the growth of the $4 \mathrm{H}-\mathrm{SiC}$ polytype. Although the presence of cerium was confirmed only by XPS measurements and only on the SiC crystallization fronts, a low Ce doping level in the bulk of
$\mathrm{SiC}\left(<10^{17} \mathrm{~cm}^{-3}\right.$; i.e. beyond the detection limit in our SIMS measurements) cannot be excluded. From our electrical properties investigations it can be concluded that the presence of $\mathrm{Ce}$ in the growth chamber influences the composition of defects in the growing $\mathrm{SiC}$ crystals. Shallow $(\approx 20 \mathrm{meV})$ defects are possibly the complexes containing the substitutional nitrogen donor and some native structural defects.

\section{Acknowledgments}

This work was supported by the Polish Research Center under grant No. 5043/B/T02/2011/40.

\section{References}

[1] Y.C. Kang, I.W. Lenggoro, S.B. Park, K. Okuyama, Mater. Res. Bull. 35, 789 (2000).

[2] G. Ren, L. Qin, S. Lu, H. Li, Nucl. Instrum. Methods Phys. Res. A 531, 560 (2004).

[3] F. Okada, S. Togawa, K. Ohta, S. Koda, J. Appl. Phys. 75, 49 (1994).

[4] A. Itoh, H. Akita, T. Kimoto, H. Matsunami, Appl. Phys. Lett. 65, 1400 (1994).

[5] J.S. Blakemore, Solid State Physics, Cambridge University Press, Cambridge 1985.

[6] G. Pensl, W.J. Choyke, Physica B 185, 264 (1993).

[7] S.W. Huh, H.J. Chung, S. Nigam, A.Y. Polyakov, Q. Li, M. Skowronski, E.R. Glaser, W.E. Carlos, B.V. Shanabrook, M.A. Fanton, N.B. Smirnov, J. Appl. Phys. 99, 013508 (2006).

[8] N.S. Saks, S.-H. Tyu, A.V. Suvorov, Appl. Phys. Lett. 81, 4958 (2002).

[9] W.J. Moore, J. Appl. Phys. 74, 1805 (1993). 